\title{
INUNDACIONES POLÍTICAMENTE CONSTRUIDAS. EL MEGAPROYECTO HÍDRICO CHONE EN ECUADOR
}

\begin{abstract}
RESUMEN
La construcción e implementación del megaproyecto hídrico Chone (Costa ecuatoriana) ha sido legitimada como medio para promover el desarrollo de la ciudad, pero ha inundado y transformado territorios rurales campesinos de manera permanente. El discurso utilitario en torno al «beneficio de las mayorías» urbanas ha justificado el proyecto, en desmedro de la presumida «minoría subdesarrollada» en el área rural. Durante la implementación del megaproyecto, los campesinos han sido presentados como «atrasados» y «pre-modernos»; alejados de los imaginarios urbanos modernos acordes con la noción oficial de progreso y desarrollo. Los hacedores de política presentan el riesgo de inundaciones sólo como un problema «natural» $\mathrm{y}$ «tecnológico», dejando ocultas las relaciones de poder que construyen las transformaciones territoriales urbano-rurales. Desde la ecología política proponemos que la (sobre) abundancia de agua es también una construcción política y no únicamente un fenómeno tecno-natural. Concluimos argumentando que los megaproyectos no sólo son medios de control de agua, sino que constituyen herramientas para ordenar y gobernar la sociedad.
\end{abstract}

Palabras clave: megaproyecto hídrico; inundaciones; políticas hídricas; discursos; Ecuador.

\section{Abstract \\ POLITICALLY-BUILT FLOODS. THE CHONE WATER MEGAPROJECT IN ECUADOR}

The construction and implementation of the hydraulic mega-project Chone (coastal Ecuador) was legitimized as a means to promote city development but has flooded and dramatically transformed rural

a Centro de Estudios y Documentación Latinoamericanos (CEDLA), Universidad de Amsterdam, Roetersstraat 33, 1018 WB Amsterdam, Países Bajos. juanhidalgo_b@hotmail.com

b Department of Environmental Sciences, Wageningen University, The Netherlands. rutgerd.boelens@wur.nl CEDLA Centre for Latin American Research and Documentation, The Netherlands.

Department of Geography, Planning and International Development Studies, University of Amsterdam, The Netherlands.

Fecha de recepción: septiembre 2018. Fecha de aceptación: diciembre 2018. 
territories. A utilitarian discourse regarding the «benefits of the urban majority» justified this project, at the expense of the ostensible «underdeveloped minority» in the countryside. Throughout the implementation of the megadam, peasants were represented as «backward» and «pre-modern», in need of adopting modernist urban imaginaries to fit official notions of progress. Policy-makers presented the risk of flooding as a mere «natural» and «technological» problem, obscuring the power relations that produce the urban-rural territorial transformations. From a political ecology perspective, we contend that the abundance of water is a political construct, instead of just a techno-natural phenomenon. We conclude by arguing that mega-projects are not only means to control water but also constitute mechanisms to order and govern society.

KEYwORDs: megadams; flooding; water politics; discourses; Ecuador

\section{INTRODUCCIÓN}

En el último siglo, en todo el mundo, los centros urbanos han llegado a consolidarse como espacios privilegiados de «desarrollo» $\mathrm{y}$ «progreso», que acogen a una gran cantidad de actores y actividades económicas (ej. Swyngedouw, 1997). Uno de los problemas más acuciantes de las urbes es el acceso al agua (Bakker, 2010). La creciente incertidumbre sobre los potenciales efectos del cambio climático (sequías e inundaciones) y la permanente migración del campo a la ciudad colocan aún mayor presión sobre la gobernanza hídrica. En muchas ciudades estos problemas no sólo giran en torno a la provisión de agua, sino también en mantener el «exceso» de agua alejado. En este contexto, la construcción de una megainfraestructura hidráulica, como trasvases y embalses multipropósito, tiene una gran aceptación entre gobiernos nacionales, planificadores de gobiernos locales y actores del sector privado (financieros y compañías constructoras) (Adams, 2009). Permiten acumular, conducir y (re) distribuir agua en función de las necesidades de la ciudad (Shah et al., 2018; Swyngedouw, 1997). De manera antagónica, el espacio rural es comúnmente presentado como el «otro», como «menos desarrollado» o «atrasado», inclusive es presentado como «tierra vacía» o «vaciable» (Boelens, 2015a; Hoogendam y Boelens, 2018; Nixon, 2010). A partir de esto, sugerimos que este antagonismo es construido (consciente o inconscientemente) desde y para los intereses de lo urbano y sus propias visiones de desarrollo. Tal construcción posiblemente parte del hecho de que la gran mayoría de ríos, áreas de recarga de acuíferos y otros cuerpos de agua dulce se originan justamente en lo rural. Es decir, la ciudad necesita legitimar su posición en el juego de poder para controlar esas aguas.

En el campo de los estudios críticos de la gobernanza del agua, las trasferencias de agua desde lo rural a lo urbano para consumo humano y saneamiento son temas prominentes. Tales trasferencias son comúnmente justificadas por el poderoso discurso sobre «escasez de agua» (Bakker, 2010; Zwarteveen y Boelens, 2014; Lynch, 2013; Riaz, 2002; Swyngedouw, 2004). De igual importancia, pero tímidamente abordada es la «sobreabundancia de agua», en especial su relación a potenciales riesgos de inundación utilizados discursivamente para 
legitimar la adopción de políticas hídricas de gran envergadura (ej. megainfraestructura hídrica: represas, trasvases). Mientras estudios críticos muestran como el discurso «naturalizador» de la escasez hídrica facilita el despojo del recurso hídrico desde territorios rurales en beneficio de áreas urbanas, la sobreabundancia emerge, casi desapercibida, como un problema que se enmarca precisamente en lo opuesto: dejando la abundancia del agua en los espacios rurales, previniendo su flujo hacia los espacios urbanos o de mayor «desarrollo».

En este artículo argumentamos que políticas, modelos y megainfraestructura de agua son justificadas a partir de la categorización de las áreas urbanas como «desarrolladas» o «con potencial de desarrollo» y a «lo rural» como un espacio «atrasado», con menos valor social, político y económico. Nuestro objetivo es analizar como este aparente antagonismo es utilizado como justificación principal para legitimar la construcción de una megapresa, y con ella una profunda transformación del territorio rural. Este artículo toma como ilustración el estudio de caso del megaproyecto multipropósito Chone, ubicado en la costa ecuatoriana. Este trabajo se realizó mediante una estancia larga de campo (5 meses) en el año 2014-2015. Se implementaron entrevistas semiestructuradas y grupos focales con campesinos, técnicos del gobierno, constructores y políticos locales. También se implementó observación participativa con registro etnográfico. Los y las entrevistados/as fueron seleccionados/as en base a revisión de información secundaria y al método bola de nieve. Con un total de cuarenta y dos entrevistas, la investigación incluyó información de actores a favor y en contra del embalse, y también «neutrales». El análisis se basó en tres ejes focales: i) análisis discursivo de legitimación y contestación en torno al megaproyecto, ii) proceso organizativo de movimiento de resistencia, y iii) estrategias gubernamentales de implementación del megaproyecto.

\section{LA SOBREABUNDANCIA DEL AGUA EN EL CONTEXTO URBANO-RURAL: CONTROL DEL AGUA Y GOBIERNO DE LA SOCIEDAD}

Los problemas de inequidad e injusticia sobre el agua son condicionados por relaciones de poder (Boelens, 2015b; Linton y Budds, 2014; Swyngedouw, 2015). Es por esto que su gobernanza está influida por intereses y contradicciones que afectan su control y acceso, tanto en las ciudades (Swyngedouw, 1995) como en los sectores rurales (Hidalgo, Boelens y Vos, 2017; Mena-Vásconez, Boelens y Vos, 2016), y en las relaciones entre ambos (Riaz, 2002; Perreault, 2014; Hommes y Boelens, 2017). El destino del agua (agua potable, riego, industria, etc.) y los medios (política pública, leyes, modelos de gobernanza, infraestructura/tecnología, etc.) utilizados para captarla, acumularla y distribuirla, están profundamente influenciados por distintas propuestas de configuración de la relación entre agua (naturaleza), tecnología y sociedad (Baghel, 2014; Swyngedouw, 2015). Para legitimar tales posiciones se construyen discursos en torno a dos condiciones extremas relacionadas con el control y acceso al agua: escase $z$ sequías, y abundancia $=$ inundaciones . 
El imaginario de la escasez de agua ha construido una base importante para legitimar acciones institucionales, económicas y tecnológicas de gran impacto socio-ambiental. Lynch, por ejemplo, muestra críticamente como el discurso de la escasez (atribuido solo a causas «naturales») es impulsado históricamente por instituciones internacionales como Naciones Unidas (UN), la Organización para la Alimentación y Agricultura (FAO) y el Banco Mundial (WB) y, adoptado por Estados y gobiernos para justificar sus acciones en torno a una «buena» gobernanza del agua. Se insiste en que «Las recomendaciones políticas de las instituciones internacionales que buscan transformar la gobernanza del agua dulce [...] están influidas por el temor a una crisis inminente causada por una absoluta escasez del recurso» (Lynch, 2013: 70). A través del caso peruano se expone cómo este discurso universalizado tiende a mostrar la gobernanza del agua como una cuestión apolítica y natural, a la vez de mantener en la penumbra aspectos esenciales sobre su construcción social, política y económica; aspectos que en el fondo definen quien, como, cuando y bajo que condiciones se accede al recurso.

Si bien el estudio crítico de la escasez del agua resulta clave para entender los problemas de injusticia hídrica y sus intrínsecas relaciones de poder (Bakker, 2003; Keil, 2005; Watkins et al., 2006; Rodríguez de Francisco y Boelens, 2015; Hommes y Boelens, 2018; Erensu, 2013), consideramos que no es el único discurso que tiende a oscurecer los intereses inmersos en su gobernanza, en especial en el contexto urbano-rural. Así como para el caso de la escasez, la sobreabundancia es relativa, la construcción social del exceso de agua se traduce muchas veces en «riesgo de inundaciones» (Osti, 2017). En esta lógica naturalizada, los organismos del estado definen cuales son áreas de mayor y cuales de menor riesgo. A partir de ello se precisan acciones para disminuir potenciales contingencias de inundación en ciertas zonas y no en otras zonas. En la definición de dichas áreas frecuentemente existe una criticable directa correlación entre: área de «mayor valor» económico corresponde a un mayor riesgo y viceversa (Vojinovic, 2015). Este es el caso de las planicies o valles de miles de cuencas hidrográficas, considerados «más valiosos» que las partes altas; o a su vez, las áreas urbanas versus las rurales. Una de las soluciones universalizadas para controlar el riesgo de inundaciones es la construcción de megaobras como embalses, cortas o desvíos fluviales en zonas rurales, ubicados generalmente aguas arriba de centros urbanos y/o centros rurales del capital (Hidalgo-Bastidas, Boelens y Isch, 2018). Tal como muestra Osti (2017) en el caso italiano, el foco de la implementación de acciones e infraestructura para prevenir el riesgo de inundaciones en estas zonas es contencioso y tiene que ver con la necesidad de encontrar «espacios rurales para ser ocupados, [...], la imagen de seguridad, y la búsqueda de un balance coste/beneficio urbano-rural» (p. 266). Este regateo entre lo urbano y lo rural sugiere que - al igual que para el caso de la escasez - la llamada sobreabundancia no siempre es por causas físicas-naturales sino que, siguiendo al discurso «riesgo de inundación de áreas valiosas», obedece a razones y decisiones profundamente sociales, económicas y políticas. En términos utilitarios, ciertos 
territorios podrían ser «sacrificables» en función del bienestar común o de la llamada mayoría que habita en aquellos territorios catalogados con un «mayor» valor (Nixon, 2010). Desde esta perspectiva la necesidad de evitar la sobreabundancia de agua como discurso puede convertirse en una herramienta poderosa que transforma políticas, instituciones, infraestructura hídrica y las formas de vida de personas y sus relaciones territoriales.

Para estudiar la sobreabundancia de agua en un contexto urbano-rural es útil adoptar una mirada crítica que vaya más allá de lo urbano o de lo rural por separado, una ecología política urbano-rural (Bakker, 2010; Swyngedouw, 2015; Hommes y Boelens, 2017). Una mirada que permita reflexionar sobre situaciones en que los intereses y fronteras de la ciudad traspasan sus propios límites geográficos. En esta perspectiva la ciudad es adoptada como referente ya que, con excepción de áreas rurales capitalistas como grandes centros agroindustriales (ej. plantaciones de banano en el caso ecuatoriano), constituyen la prioridad social, política y económica en donde recaen los imaginarios de modernidad y progreso de los gobernantes. En tanto «lo rural» es comúnmente percibido como un espacio atrasado (ej. Boelens, 2015). Con esta aproximación no asumimos que la relación urbano-rural o campo-ciudad es unidireccional, por el contrario, su influencia es bilateral. Lo que sí explicitamos desde este enfoque es que el nexo entre estos dos espacios es inequitativo y que obedece a relaciones de poder que históricamente han ubicado a los centros urbanos como referentes de desarrollo, mientras lo rural ha sido relegado a ser un espacio de sacrificio (Nixon, 2010).

Desde esta perspectiva, el campo ha caminado por la cuerda floja de lo diferente y al mismo tiempo de lo potencialmente igual. Es diferente por su condición de atraso con respecto a la ciudad, pero con potencialidad de volverse igual mediante la adopción de valores e identidades imaginados por aquellos que se encuentran en el poder. Estos modelos fundamentados generalmente en nociones dominantes de expertos y tecnócratas de la ciudad crean su propio mundo hídrico moldeado desde sus propias necesidades, posiciones, conocimientos e intereses; que no sólo tienen efecto sobre el agua, también sobre la sociedad. Por tanto, nuestro entendimiento de las relaciones agua-sociedad van más allá de la concepción de los seres humanos gobernando el agua, sino se extiende a seres humanos gobernando la sociedad a través del agua (Boelens, 2015a: 13; ver también Zwarteveen, 2015).

\section{El «RETORNO» DEL ESTADO: LA REVOlUCión CIUDADANA DEL BUEN VIVIR} Y LA MODERNIDAD

El «retorno» del estado en Ecuador estuvo acompañado por la jerarquización de conocimientos, formas de gobernar, territorios, personas y espacios geográficos. En el año 2007 un nuevo gobierno asumió el poder. Su bandera de lucha fue refundar la patria con una revolución con los ciudadanos. Una revolución que, según el entonces presidente Rafael Correa, dejaría «la larga y triste noche neoliberal» atrás. Guiado por esta premisa y bajo un 
discurso progresista de justicia social, igualdad y soberanía, el gobierno de la llamada revolución ciudadana emprendió varios esfuerzos para fortalecer el rol y la presencia del estado que fue desmantelado en el pasado por el neoliberalismo y sus consecuentes crisis sociales, políticas y económicas. Parte del itinerario de este cambio de época fue emprender una profunda modernización del estado, ordenar el caos en el que estaba sumido el país, recuperar la institucionalidad lacerada y llevar desarrollo en forma de buen vivir a cada rincón de la patria. Buen vivir recoge elementos de las cosmovisiones de pueblos y nacionalidades indígenas, combinándolos con perspectivas de vida y de políticas progresistas. Sin embargo, es preciso señalar que a pesar de la adopción de esta visión de desarrollo alternativa, el gobierno progresista la vació de contenido crítico y le incluyó una perspectiva utilitarista del Estado, mezclado con bases del propio capitalismo (Van Teijlingen, 2016; Valladares y Boelens, 2017). Contrario a la visión alternativa planteada por este concepto, el gobierno profundizó una agenda extractivista, bautizada por Eduardo Gudynas como «neoextractivismo» (ver Gudynas y Acosta, 2010).

Entre los esfuerzos más sobresalientes para lograrlo cuenta la alianza con otras fuerzas políticas (movimiento indígena, academia crítica, campesinos, etc.) y ciudadanas para construir en el 2008 una nueva carta constitucional garantista de derechos (Becker, 2012), como ninguna otra. En esta lógica la importancia de la participación ciudadana, el reconocimiento de la plurinacionalidad, la introducción amplia del acceso al agua como derecho humano y el respeto a los derechos de la naturaleza son ejemplos de este giro plasmado en la nueva constitución política.

A pesar de haber iniciado con propuestas bastante progresistas e inclusivas, con el pasar de los años el proyecto político fue recibiendo críticas incluso desde grupos que inicialmente lo apoyaban (Becker, 2012). Estas críticas han estado fundamentadas principalmente en las contradicciones entre el discurso y la práctica gubernamental. Principalmente la crítica giró alrededor de la incapacidad del gobierno para incluir a los actores que piensan diferente, a la criminalización de la protesta (Amnistía Internacional, 2012), al autoritarismo, a la falta de independencia de los poderes del estado, a la deslegitimación y consecuente debilitamiento de la organización social autónoma (Acosta et al., 2013) y a la imposición de su verdad como la única y superior a otras posturas. Esta tensión y críticas salen a flote particularmente en el desarrollo de los llamados megaproyectos hidráulicos multipropósito.

Uno de los ejes centrales del proyecto político fue el cambio de la matriz productiva y energética, para lo cual el manejo tecnocrático del agua tuvo un rol protagónico. Como parte de la nueva institucionalidad para impulsar este enfoque y el de otros recursos definidos como recursos estratégicos se crearon, el Ministerio de Sectores Estratégicos (MICSE), la Secretaría Nacional del Agua (SENAGUA) y la Empresa Pública Ecuador Estratégico (EEEP). El primero dirigía la política rectora sobre el manejo de los recursos estratégicos, la segunda dictaba política nacional sobre gestión del agua y construía grandes proyectos hídricos, mientras 
que la tercera realizaba proyectos de compensación económica a las comunidades afectadas por la construcción e implementación de proyectos estratégicos nacionales a partir de la renta petrolera y minera. A pesar del discurso progresista y participativo, el diseño, la construcción e implementación de megaproyectos han sido fuertemente contestados por parte de las comunidades locales afectadas. Contrariamente a lo esperado por un gobierno de este tinte, estos proyectos han sido acompañados por violencia estatal, desalojos forzados, falta de participación en procesos de toma de decisión, desconocimiento de la consulta previa y elaboración de estudios de impacto ambiental cuestionados (Acosta et al., 2013; Amnistía Internacional, 2012).

En este contexto, a la vez que desde el estado se legitimó un estereotipo dominante de desarrollo o buen vivir, casi por defecto se deslegitimaron otras formas de desarrollo y territorialidad, en especial aquellas opuestas a las obras y acciones del proyecto político oficialista (Boelens et al., 2015; Van Teijlingen, 2016). Tal como lo expresó el entonces presidente Correa durante el discurso inaugural de un embalse construido por su gobierno: «Un saludo especial a las familias afectadas por el embalse, que con patriotismo colaboraron sabiendo que esto era en función del bien común. Se sacrificaron [...], hicieron un poco de esfuerzo y creo que esos esfuerzos han tenido su justa compensación; han sido reasentadas, ahora cuentan con una casa digna, de cemento». ${ }^{1}$ Implícitamente sugería que gracias al alineamiento de la gente al proyecto de desarrollo oficialista tenían acceso a un vivienda digna, a diferencia de un pasado neoliberal en el que vivían de manera indigna.

\section{El Multipropósito Chone y el sueño de controlar las inundaciones}

La ciudad de Chone está ubicada en la costa ecuatoriana y es la cabecera urbana del cantón homónimo. ${ }^{2}$ Es una ciudad de mediano tamaño que ocupa el 0,5 \% de la superficie cantonal con cerca de 52 mil habitantes. ${ }^{3}$ Tal como se aprecia en la figura 1 la mayoría de la población se distribuye en las áreas rurales que ocupan más del 99 \% de la superficie cantonal. Es decir, las mayorías están ubicadas en el espacio rural. Además, alrededor del 70 \% de la población depende de la agricultura o ganadería consolidando el campo como el principal espacio de ocupación socioeconómica del cantón (INEC, 2010).

1 Comunicación personal, 27 Jun 2013.

2 La división político-administrativa del Ecuador se constituye de cuatro niveles respectivamente subordinados: provincia, cantón, parroquia y barrios (urbano) o, recintos y comunidades (rural).

3 Datos sistematizados a partir del Censo de población y vivienda 2010. 


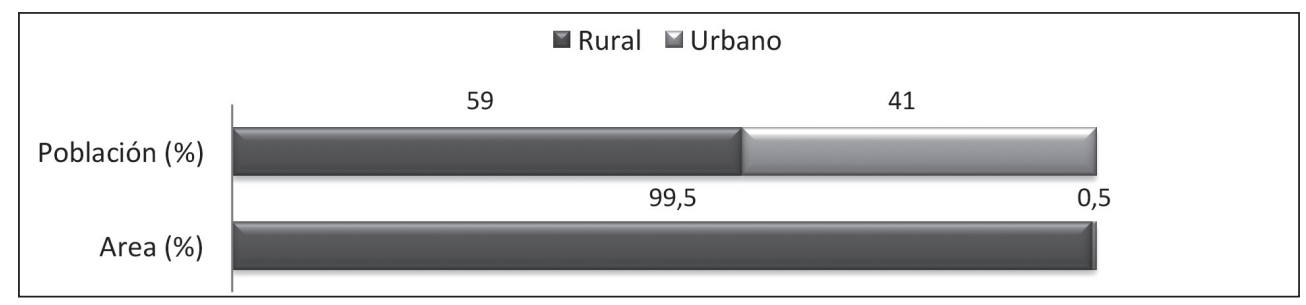

Figura 1. Distribución de la población en el espacio rural y urbano en el cantón Chone.

Fuente: INEC, 2010; adaptación de los autores.

Una característica particular de esta ciudad es que se asienta en la parte más baja del valle a orillas del río Chone. Esto ocasiona que durante cada época lluviosa (desde diciembre a mayo) la ciudad sufra inundaciones por el desbordamiento del río Chone y sus principales tributarios: río Grande, río Garrapata y río Mosquito (mapa 1). Es por esta razón que desde inicios de los años 80 se han venido planificando alternativas de infraestructura para controlar las inundaciones de la zona urbana. Junto a un canal de desagüe, una megarepresa de 59,5 m de altura y 113 millones de $\mathrm{m}^{3}$ sobre el río Grande fue construida como parte del proyecto multipropósito que fue inaugurado por el expresidente Correa en noviembre del 2015. El proyecto fue promocionado por sus múltiples fines que podrían eventualmente mejorar la provisión de servicios como riego a zonas bajas (alrededor de $2.200 \mathrm{ha}$ ) y agua potable para la ciudad.

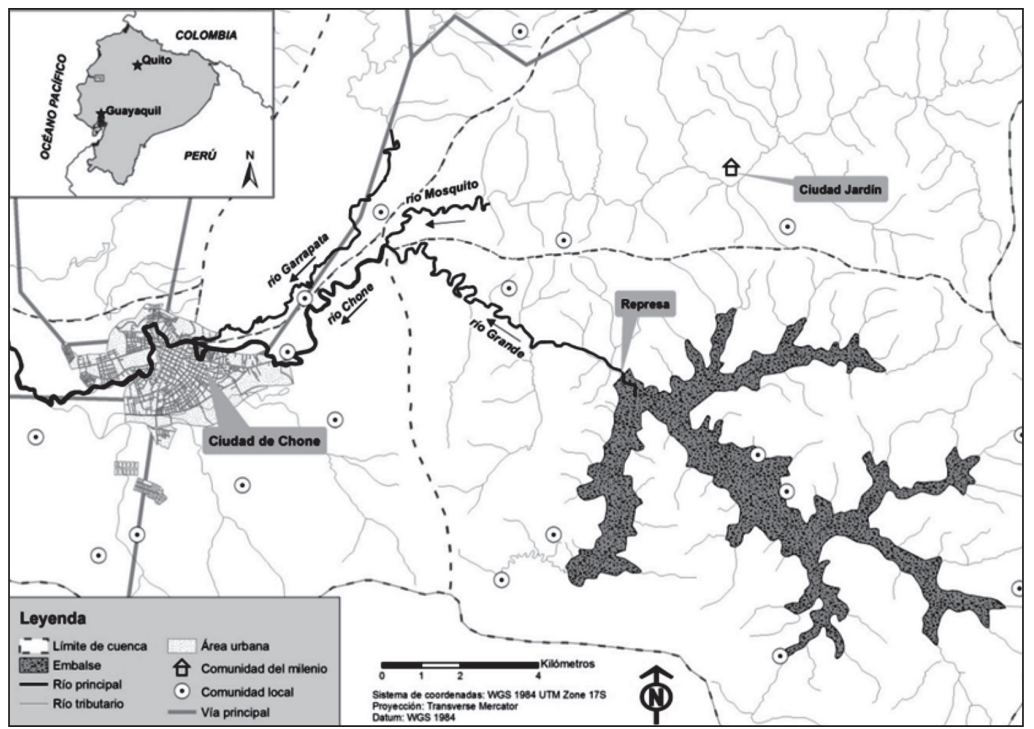

Mapa 1. Ciudad de Chone y los principales ríos que drenan sus aguas en el valle. 


\subsection{Justificando la represa en Chone: énfasis en el riesgo/desarrollo urbano}

A pesar del predominio y relevancia socioeconómica de lo rural, desde las primeras discusiones en torno al proyecto, la ciudad de Chone se construyó como un espacio de privilegio frente a lo rural. Parte de este posicionamiento involucró fortalecer el discurso en torno al riesgo de las inundaciones y desarrollo centrado en la imagen de la ciudad. Mientras el primer aspecto fue el justificativo más importante, el segundo tuvo un rol destacado sobre todo en la creación de una imagen del «otro», de lo rural como menos desarrollado o atrasado. Así lo resaltaba el diario de mayor circulación nacional en noviembre de 1979, cuando por primera vez el tema de las inundaciones se presentó como un evento trascendental:

«Atenderán pedidos del cantón Chone: Se realizará una reunión ampliada en el CRM. ${ }^{4}$ Por su parte el gobernador de la provincia manifestó que el desvío del río Chone era la obra de mayor importancia, pues si se demora en solucionar el asunto, esta ciudad podría verse inundada nuevamente en el invierno». ${ }^{5}$

Esta urgencia llevó a que se realicen estudios durante casi tres décadas con el fin principal de salvaguardar los intereses de la urbe. La reactivación definitiva del proyecto tuvo lugar en febrero del 2008 después de una nueva temporada invernal que anegó toda la ciudad y gran parte del valle del río Chone. El ex presidente Correa, le dio el impulso definitivo que necesitaba el proyecto:

«Aquí la solución definitiva es el proyecto múltiple Chone que solo por el control de inundaciones ya tendría razón de ser [...], vamos a hacer ese proyecto!» ${ }^{6}$

Enseguida después de la orden presidencial el Gobierno Provincial de Manabí contrató la actualización de los estudios que consistió básicamente en su digitalización y actualización de costos. ${ }^{7}$ No incluyó actualización ni ampliación de aspectos técnicos. La urgencia de controlar las inundaciones era tal que el gobierno desplegó un gran contingente propagandístico durante la fase de promoción del proyecto. Colocó en toda la ciudad gigantografías que recordaban inundaciones de la ciudad (Figuras 2 y 3). Por otro lado, los reclamos y argumentos de las comunidades en río Grande, que hasta entonces habían permanecido al margen de la planificación oficial, fueron subestimados. Rumores extraoficiales era toda la información con la que contaba la gente que habitaba el área rural en donde se implementaría la represa.

4 Centro de Rehabilitación de Manabí era la entidad regional a cargo de la planificación y gestión de los recursos hídricos de la provincia hasta el 2009.

5 El Comercio, 26 Noviembre 1979.

6 Rueda de prensa, 24 Feb 2008. https://www.youtube.com/watch?v=6qS8OtRWd-o.

7 El Ecuador tiene tres niveles regionales de gobierno: provincial, cantonal y parroquial. 


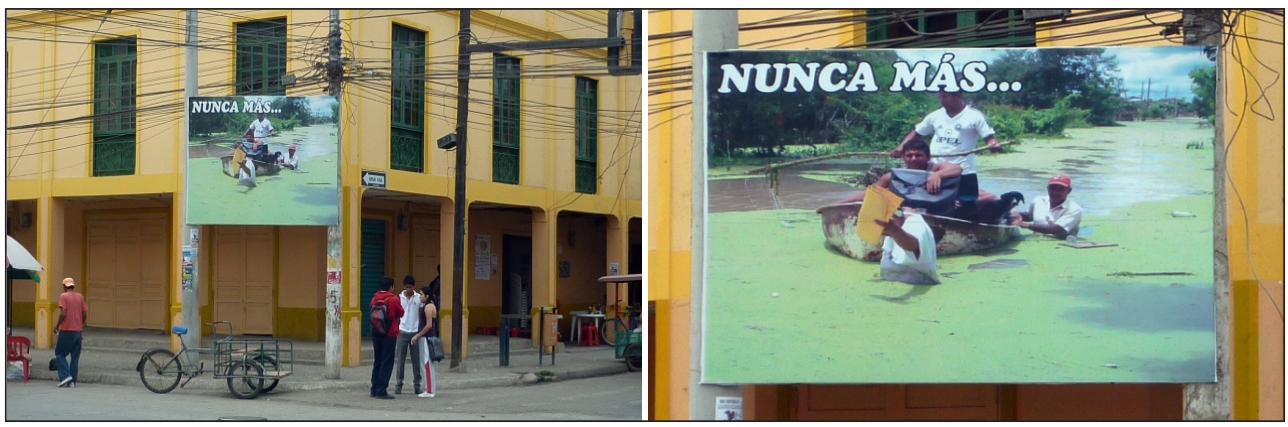

Figuras 2 y 3 . Gigantografía en una esquina de la ciudad de Chone.

La justificación de la obra como prioritaria para la ciudad y la subestimación de las áreas rurales afectadas se reflejan en lo extemporáneo del Estudio de Impacto Ambiental (EIA) y del proceso de participación social. Tal como manifiesta un campesino afectado: «el punto de ellos [SENAGUA] es hacer el embalse aquí y de ahí recién ver cómo nos van a reubicar... no tienen un plan...». ${ }^{8}$ Esto es ratificado por un informe interno de la misma SENAGUA (2012) en donde se ratifica que el proceso de licencia ambiental y participación social tuvo lugar casi cinco meses después de haber firmado el contrato de construcción del proyecto.

La justificación de la obra alcanzó incluso niveles de indiferencia frente a lo rural. Así lo expresa uno de los técnicos del área social y ambiental del proyecto al reconocer que la construcción de la obra era un imperativo: «lo que no concebíamos [durante los proceso de participación social] era que la construcción de la represa se consensuara porque es que ahí no había nada que consensuar, eso iba porque iba. [Además], esas cosas técnicas las puedo socializar con ingenieros, con academia, pero la comunidad no lo entienden». ${ }^{9}$ De esta forma la justificación del proyecto y la subordinación de lo rural (en particular la zona afectada por la represa) fueron de la mano.

El multipropósito estaba ampliamente justificado y legitimado por gran parte de habitantes de la ciudad. Tal como lo reconoce un asesor de la SENAGUA: «la población urbana apoyaba totalmente el proyecto. En la zona rural en río Grande estaban en desacuerdo». ${ }^{10}$ Inclusive a lo largo de la vía que conducía a la zona del proyecto se leía en los letreros: «Aquí se construye el sueño de Chone. El cambio ya está en marcha y nada lo detiene». El contrato de construcción se firmó en julio del 2010 con la presencia de autoridades del gobierno central y local. En ese evento los habitantes de las comunidades de Río Grande tomaron los alrededores del lugar donde se realizaba el acto y, aunque se habían presentado protestas con

8 RTU Noticias, 16 Agosto 2011. https://www.youtube.com/watch?v=Cg2kpmFHrF8.

9 Comunicación personal. 1 Septiembre 2015.

10 Comunicación personal. 2 Agosto 2015. 
anterioridad, desde ese día lo rural, como diferente, empezó a ser considerado también como potencialmente igual.

\subsection{Las comunidades de Río Grande son «diferentes» pero «potencialmente iguales»}

«Querer transformar [la sociedad] vía política pública y vía eficiencia, eficacia no es posible, porque la transformación verdadera está en el ser humano: debemos decodificar patrones pre-modernos y codificar nuevos patrones modernos para la construcción del Buen Vivir» (Exministro de la Secretaría Nacional del Agua, 21 Julio 2016).

Tal como lo expresa el exministro, la construcción del desarrollo o Buen Vivir como objetivo transversal del gobierno, estaba basado en una dualidad necesariamente antagónica: lo «pre-moderno» y lo «moderno «. En la práctica esto se evidencia en el proceso de construcción de la ciudad de Chone como un espacio superior para ser protegido de inundaciones, mientras que la imagen de lo rural, (consciente o inconscientemente) fue subordinada como el otro, lo atrasado. No obstante, esta caracterización de lo rural a la vez permitió al oficialismo la legitimación del proyecto como un contenedor de beneficios para los campesinos afectados por el embalse. Es decir, los afectados podían eventualmente volverse desarrollados, tal como en la ciudad: se convirtieron por lo tanto en potencialmente iguales. Así lo afirma un técnico del área socioambiental:

«Ellos ya toda la vida han vivido aislados. Claro que esto no se les puede decir delante de ellos porque lo entenderían como una ofensa, porque pensarían que nosotros [los técnicos] los hacemos de menos.[...] Nosotros hemos sido generosos en intervenir. El proyecto ayudó más bien a que mejoren su vida. Ellos siempre han vivido mal, como quien dice han sido comunidades algo atrasadas».

Tal como anticipamos, la puesta en marcha del embalse no pasó sin contestación por parte de las comunidades de río Grande. Tan pronto inició el proceso de promoción y construcción esta empezó a ser criticada por comunidades donde se ubicaría el eje de la presa. La resistencia de las comunidades empezó a organizarse desde el 2009. La base social de la resistencia fue el Comité Central de Comunidades Campesinas de Río Grande (CCRG). La mayoría de sus miembros eran pequeños y medianos campesinos y propietarios de fincas ubicadas en la zona de Río Grande. Este espacio también estaba conformado por otros actores que no pertenecían al CCRG, como académicos críticos, ONGs, políticos locales y nacionales, campesinos afectados por otros embalses y ciudadanos críticos de la ciudad de Chone. La resistencia movilizó su reclamo mediante varios medios: demandas judiciales, bloqueo de calles en la ciudad de Chone y Quito, manifestaciones simbólicas de rechazo a la represa, artículos de prensa, redes sociales y prohibición de entrada a la zona de Río Grande a personas ajenas al lugar. Todas estas actividades aplazaron el inicio de la obra y físicamente evitaron la entrada de la empresa constructora a la zona. 
Este proceso de resistencia hizo que la construcción de la represa se retrasase poco más de un año; sin embargo, paulatinamente fue desmovilizada por varias acciones estatales. El movimiento de oposición se negaba a abandonar el lugar donde estaban agrupados. Era la finca de uno de los líderes, ubicada precisamente en el emplazamiento elegido para construir el embalse. Primero la SENAGUA declaró varias de las fincas como propiedades de utilidad pública. Luego un juez de Chone ordenó a la fuerza pública dar apoyo y garantías para la ocupación inmediata y violenta del predio donde se agrupaba la resistencia. Tal como se relata en el Informe Policial del operativo de octubre del 2011:

«Con el apoyo del personal policial...se procedió a ingresar a dichos predios para la ocupación inmediata ordenada por la autoridad competente al tener un numérico superior a los opositores, se les pudo disuadir y hacer abandonar los terrenos con el uso progresivo de la fuerza». (SENAGUA, 2012: 267)

Horas más tarde después del desalojo, mientras la maquinaria de la empresa constructora entraba a la zona para derribar las viviendas y desmontar la vegetación, el uso de la fuerza fue legitimado en Quito y el poder soberano del Estado fue sellado por el presidente Correa al firmar el Decreto $\mathrm{N}^{\circ}$ 914, mediante el cual se declaró a la zona de afectación del proyecto como área de seguridad nacional. El área pasó a ser resguardada por la fuerza pública lo cual permitiría garantizar la implementación de la obra. La estrategia coercitiva logró su objetivo, así lo presenta la SENAGUA (2012: 247): «Es necesario resaltar el logro obtenido con la incursión [policial] a la zona de construcción del cuerpo de la presa... la que dio como resultado la adhesión de los propietarios de la zona de Río Grande que se encontraban en oposición».

Aquellas personas que se oponían eran tachadas de radicalmente antagónicos al desarrollo o Buen Vivir. El vicepresidente incluso los calificaba de atrasa-pueblos. O como se refiere el ex ministro de SENAGUA sobre el tema: «Es una lucha entre el cambio y la tradicionalidad. [...] Los campesinos no entendían el futuro y nosotros queríamos hacerles ver el futuro. Ellos defienden formas de vida premodernas». ${ }^{11}$ Desde la visión oficialista, la oposición defendía formas de vida irracionales, diferentes a las deseadas o recetadas por el gobierno como adecuadas.

En ese contexto, varias ofertas de compensación les eran ofrecidas a los campesinos en resistencia basadas en la idea de «urbanizar la ruralidad». La propuesta incluyó la construcción de una comunidad modelo, la llamada comunidad del milenio, nombrada «ciudad jardín», y una escuela del milenio. Tal como lo expresaba un asesor de la SENAGUA: «Esto fue un gran logro, a través de la realización del multipropósito se tuvo la posibilidad de contar con una propuesta modelo de ciudad como la que se construyó». Los opositores que se acogían a las reglas, valores e ideología del gobierno respecto a lo que debe ser desarrollado, en este

11 Comunicación personal, 21 Jul 2016. 
caso lo rural bajo las reglas gubernamentales, se les abría la oportunidad de ser un poco más iguales.

4.2.1. Gobernando la sociedad a través del agua: Ecuador Estratégico EP y su sueño de una ruralidad urbanizada

Las compensaciones que el gobierno implementó tenían dos fines principales, el primero dispersar la resistencia y segundo promover el desarrollo de los afectados por la represa. En este proceso la EEEP desempeñó un papel importante. Creada en el 2011 surgió como una propuesta institucional con el fin de materializar el discurso de desarrollo y buen vivir a través de realizar inversiones como escuelas y comunidades modelo y vías asfaltadas en zonas afectadas por grandes proyectos considerados estratégicos.

En ese marco, el megaproyecto debía ser entendido como ayuda para los atrasados (diferentes) a volverse desarrollados (iguales). Bajo esta intención se planificó y construyó la comunidad del milenio para instalar una forma de vida desarrollada dirigida a los trabajadores de las propiedades afectadas. Durante la etapa de planificación de la comunidad del milenio los técnicos se acercaban individualmente a campesinos opuestos al proyecto para ofrecerles casas con la condición de que persuadieran a más gente a abandonar la protesta. Esta estrategia fue efectiva en tanto varios campesinos la aceptaron. Así lo comenta un ex dirigente opositor y primer dirigente de la comunidad del milenio:

«Nosotros nos oponíamos...pero una noche un ingeniero de SENAGUA me llamó y me dijo: "sabe el presidente les va a construir un pueblito. Tal fecha traiga toda la gente para que firmen para darles las casas”. Entonces ahí me fui y me pasé de la oposición a favor del gobierno. Nosotros cedimos. Nosotros estábamos ciegos y otros de la oposición que ahora viven aquí en estas casas también».12

Un aspecto que en gran medida guió las decisiones tomadas acerca del lugar de ubicación del reasentamiento de las familias afectadas fue la idea de que la microcuenca en donde se ubica el embalse debe ser un espacio vacío o vaciable. ${ }^{13}$ Durante las primeras etapas de planificación de las medidas compensatorias se contemplaba crear tres nuevos reasentamientos en los sitios mismos de afectación (mapa 2).

12 Comunicación personal 10 Dec 2014.

13 De acuerdo a la SENAGUA 181 familias fueron afectadas directamente por la construcción de la represa; sin embargo, la comunidad del milenio solo tiene capacidad para 81 . Es decir, cerca del $55 \%$ de los afectados no han sido reubicados. 


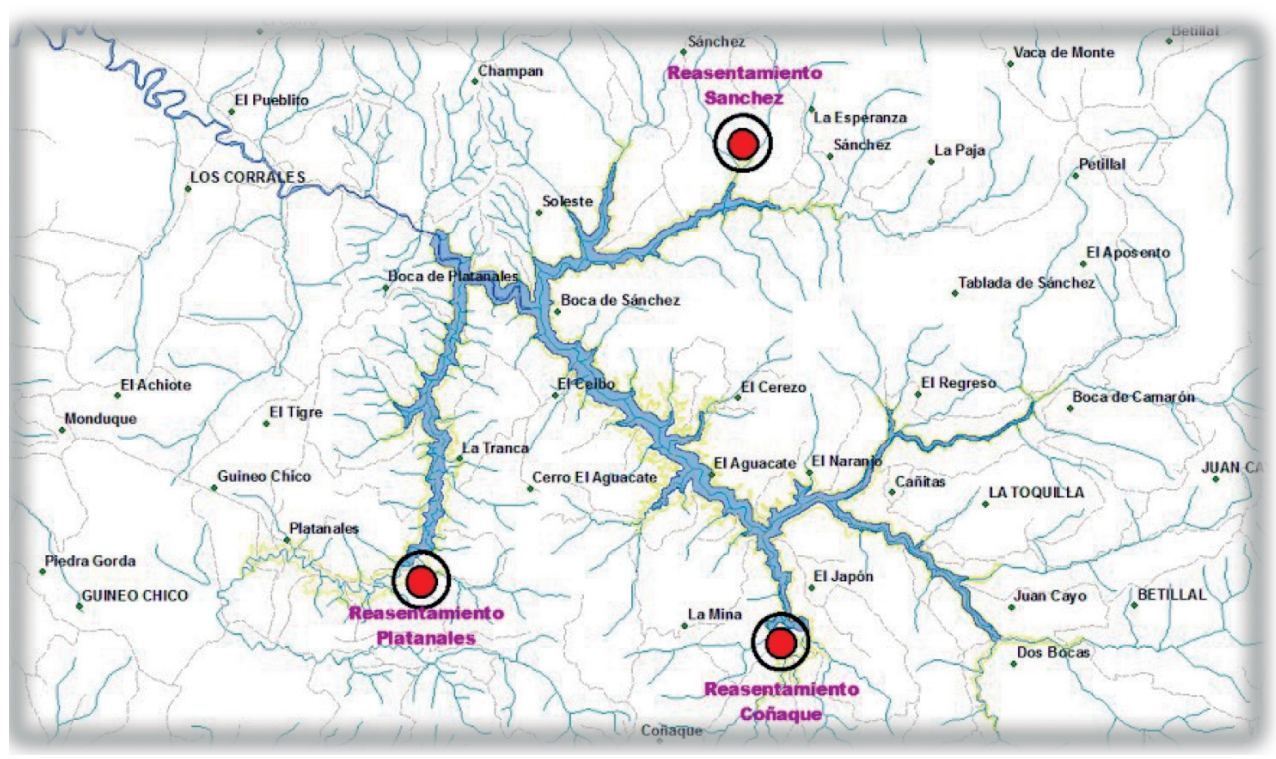

Figura 4. Plan de reasentamiento original. Fuente: SENAGUA

Sin embargo, los técnicos vieron como una oportunidad la construcción del proyecto para traer orden a ese territorio. Tal como lo expresó uno de los técnicos a cargo del manejo socio-ambiental del proyecto:

«Lo ideal es tener esto vacío de gente, [...] se decidió hacer el reasentamiento fuera de la microcuenca para evitar afectar a la calidad del agua del embalse, por el tema de los desechos o básicamente por problemas ambientales. El presidente [Correa] quería que este fuera un modelo de reasentamiento en América Latina, vinculado con que el megaproyecto fuera un modelo». ${ }^{14}$

Así es que efectivamente la comunidad del milenio se construyó fuera de la microcuenca del río Grande. Se la ubicó en la del río Mosquito (mapa 1). Esto muestra como el multipropósito no era únicamente un medio para controlar la naturaleza (al agua), pero también como fue un medio para ordenar lo social. Una vez construida la comunidad modelo, la EEEP estableció y socializó nuevas reglas y normas para vivir acorde a la nueva configuración del espacio físico. La comunidad fue construida como un ejemplo de Buen Vivir, netamente relacionado con lo urbano. Siguiendo esa lógica la comunidad — similar a cualquier ciudadela urbana - está organizada en manzanas, con señales de tránsito y límites de velocidad cada

14 Comunicación personal 1 Sept 2015. 
10 m. También cuenta con espacios comunitarios: parque, mercado, iglesia, centro de cómputo. Sin duda, muchas familias se sentían a gusto en este nuevo contexto.

Entre las nuevas reglas de convivencia de la comunidad estaban: organizarse por bloques para mantener los espacios comunes, la prohibición de hacer fiestas pasada la media noche y tener animales de corral o cerdos y no dejar inhabitada la casa por más de 15 días, de lo contrario, la EEEP analizaría la posibilidad de otorgarle la vivienda a otra familia. ${ }^{15}$

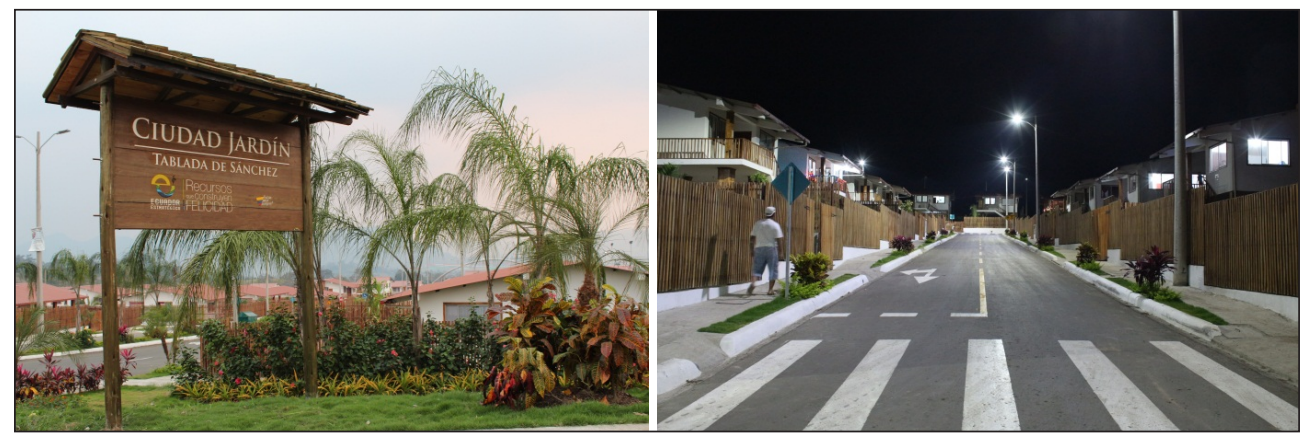

Figuras 5 y 6 . Comunidad del milenio «ciudad jardín».

Ninguna casa tiene un terreno extra para cultivar ni criar animales, en su lugar el gobierno adquirió dos fincas para promover el trabajo colectivo de los reasentados. Lo cual iba en contra de las prácticas individuales de trabajo en la agricultura que los campesinos habían practicado por siglos (Ferrín-Schettini, 1986).

A pesar de los esfuerzos del gobierno, la implementación de reglas de convivencia no fue suficiente para adaptar a los campesinos a una vida más urbana y racional. Como parte del proceso la EEEP desarrolló un juego de mesa con el fin de enseñar a los reasentados el camino adecuado — de acuerdo al proyecto político oficialista - hacia la felicidad o el buen vivir. Este juego fue implementado en la comunidad a través de promotores comunitarios, varias sesiones de capacitación y visitas de los reasentados a otros proyectos estratégicos (agrícolas, trasvases de agua, embalses, centrales hidroeléctricas) construidos por el gobierno. Los promotores comunitarios son habitantes de la localidad que aceptaron formar parte de este programa de EEEP. Siguieron un proceso de capacitación de varios meses. Su objetivo era informar a los demás habitantes del lugar acerca de los beneficios que tenían todos los proyectos estratégicos del gobierno como minería, hidroeléctricas, embalses multipropósito y extracción petrolera. Entre las actividades de los promotores comunitarios estaba la imple-

15 Esta información está basada en entrevistas con los habitantes de la comunidad del milenio y oficiales del gobierno. 
mentación tres versiones distintas del juego. Cada versión es dedicada a cada sector estratégico: minería, hidrocarburos y megaproyectos hidroeléctricos. La lógica del juego es similar a la del popular juego de mesa Monopoly. Incluye colores, dibujos y cartillas informativas en lenguaje sencillo y directo, incluye dados y una trayectoria a seguir llena de casilleros que premian o castigan en base a la elección del jugador. En tanto el jugador caiga en casilleros correspondientes a obras emblemáticas del gobierno de la revolución ciudadana, el camino a la felicidad y el buen vivir está garantizado. Parte fundamental del juego conforme avanza el jugador en el tablero, es contestar una serie de preguntas que abiertamente promueven el proyecto político gubernamental. Así una cartilla pregunta ¿En Ecuador, las actividades petroleras protegen los recursos hídricos?, la respuesta es 'Sí'; otra menciona ¿Para que se utiliza el dinero proveniente de los recursos estratégicos? La respuesta categórica es 'Para generar desarrollo al país` y tal vez una de las más sugestivas es ¿qué significa el petróleo para el Ecuador? La única respuesta es 'Desarrollo, prosperidad y bienestar'. Así, las normas y verdades del gobierno son presentadas a los habitantes de la comunidad, los nuevos iguales, en una forma sutil y lúdica.

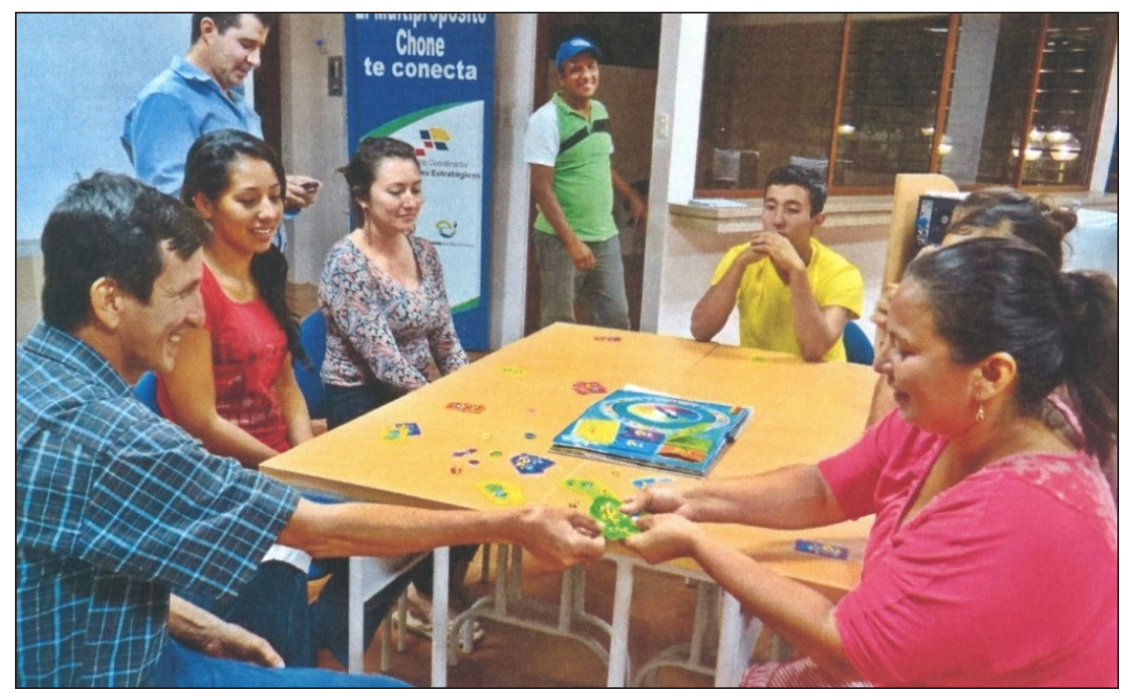

Figura 7. Habitantes de Ciudad Jardín y promotores comunitarios jugando bajo la supervisión de un técnico de Ecuador Estratégico EP. Fuente: Boletín de prensa 455 Ecuador Estratégico, 4 de agosto 2015. 
Las intervenciones de la EEEP fomentaron una diferenciación social entre campesinos reasentados y no reasentados. Varios funcionarios del gobierno, habitantes de Chone y los propios reasentados manifiestan que la forma de vida de los segundos es menos digna y moderna que la de los primeros. En otras palabras, aquellos que no se opusieron al proyecto o que cedieron a las presiones y ofertas gubernamentales ahora están, cómo en el juego de mesa, más cerca del buen vivir y la felicidad. Es así que a través de las normas y discursos vinculados al embalse, no solo el paisaje y su ecología han sido reconfigurados, también las relaciones sociales, agro-productivas y laborales de las comunidades locales. La gobernadora de Manabí, en su discurso de inauguración, no pudo expresar de mejor manera lo que representa esta megaobra y proceso de igualdad:

«Esta obra tiene alma y cuerpo. El cuerpo es esta majestuosa represa, pero el alma se está sembrando en la consciencia, en el alma de los más puros. En el alma de nuestros niños y niñas. Esta obra no solamente es infraestructura». ${ }^{16}$

En la actualidad, aunque el embalse es operativo, las inundaciones en la ciudad de Chone y sus alrededores continúan, en especial por el río Garrapata y otros tributarios. No obstante, más de 1.000 ha, que no se inundaban antes del proyecto, fueron permanentemente anegadas en la zona de río Grande. Además del impacto ambiental causado, cerca de una centena de familias campesinas no han sido reasentadas, y muchas han quedado en condiciones aún más precarias. Varias afrontan dificultades de transporte por la presencia acumulada de lechuguín (lirio de agua) en el espejo del embalse y el incremento significativamente del coste de desplazamiento. Además se evidencia una coerción de las relaciones sociales preexistentes. Así, como caballo de Troya, la megaobra se revistió de técnica a la vez de tener un alma que impulsa, legitima y justifica el proyecto político oficialista que realza paradojas entre lo rural y urbano, y la gobernanza de la abundancia de agua.

\section{DisCUSIÓN Y CONCLUSIONES}

En este artículo hemos presentado como el discurso de sobreabundancia de agua es utilizado para promocionar megaembalses como remedios técnicos y apolíticos, que a la vez exacerban relaciones de inequidad entre lo urbano y lo rural. Mostramos cómo la solución ingenieril escogida en Chone obedece a una política nacional de creencia en lo moderno como un peldaño hacia el Buen Vivir. Las reformas estatales, proclamadas bajo consignas de participación e inclusión, estuvieron profundamente informadas por una jerarquización de conocimientos (moderno vs. premoderno), formas de gobernanza (buena vs. tradicional), ideologías (socialismo del buen vivir), territorios (desarrollo vs. atraso), personas (mayorías vs. minorías sacrificadas) y espacios geográficos (urbano vs. rural). Bajo estas circunstancias

16 Comunicación personal 24 Nov 2015. 
la ciudad de Chone fortaleció su imagen como un territorio de desarrollo donde supuestamente habitan las mayorías, mientras río Grande y sus comunidades rurales fueron designadas con la etiqueta de atrasadas y sacrificables. Esto ha llevado a que, tras la construcción de la represa, en la actualidad la ciudad permanezca parcialmente protegida de inundaciones, ya que las aguas del río Grande han sido controladas. Gran parte de la ciudad y sus alrededores siguen inundándose por el desborde de otros tributarios como el río Garrapata. El problema que motivó inicialmente la construcción del multipropósito para controlar las inundaciones lo que en realidad ha hecho es inhabilitar (inundar) de manera permanente cientos de tierras campesinas.

El caso de Chone permite cuestionar el discurso utilitarista de las llamadas mayorías versus el de las consideradas minorías. Mostramos como en el contexto estudiado, lo rural es el espacio en donde habita literalmente la mayoría de la población. Comúnmente megaembalses como el de Chone son justificados bajo esta falsa premisa utilitaria: el bienestar de las mayorías; sin embargo, aquellas minorías son en realidad las mayorías. Es así que el mito utilitario, más que a hechos objetivos, frecuentemente obedece a una jerarquización conveniente, a los intereses de quien ostenta el poder.

El artículo demuestra como esa construcción jerárquica no solo justifica la construcción de megaproyectos como el de Chone, también - y de manera estructural- (re)produce afectaciones y transformaciones profundas en territorios rurales y sus formas de vida. Tal como presentamos, las llamadas minorías señaladas bajo la etiqueta de sacrificables e incluso pre-modernas pueden ser sujetos de transformación. En esa medida y siguiendo normas, valores, ideologías y formas de vida predesignadas por aquellos en poder, las minorías pueden ser tan iguales como los que gozan ser parte de las mayorías del poder. Es decir, los rurales pueden ser tan desarrollados, tan modernos y tan felices como los urbanos.

El caso de la sobreabundancia de agua presentado enfatiza en que el nexo entre lo rural y lo urbano no únicamente se problematiza por la extensión de los límites de la ciudad hacia el campo (Hommes y Boelens, 2017, 2018). Complementariamente, tal extensión se reproduce por desencuentros entre formas de categorización distintas y opuestas, otorgadas históricamente a los espacios urbanos y rurales. De esa manera, la sobre-abundancia no es un fenómeno natural y climatológico: el análisis acerca de la construcción política de la abundancia del agua y los riesgos de inundación en contextos urbano-rurales desvela formas particulares de injusticia hídrica. En particular nos permite entender, desde una lectura crítica de zonas inundadas por megaembalses, como las aguas rurales no son únicamente controladas en su materialidad, pero también son la ventana para desvelar formas de reordenamiento social.

\section{ReFERENCIAS}

Adams, W.M. (2009): Green Development. Environment and sustainability in a developing world. Routledge, London y New York. 
Acosta, A., Cuvi, J., Ospina, P., Unda, M., Torre, C. de la, Villagómez, G., .. Machado, D. (2013): El correísmo al desnudo. Abyayala, Quito.

Amnistía Internacional (2012): Para que nadie reclame nada ¿Criminalización del derecho a la protesta en Ecuador? Amnistía Internacional, Madrid.

Baghel, R. (2014): River Control in India: Spatial, Governmental and Subjective Dimensions. Springer, Dordrecht.

Bakкer, K. (2003): Archipelagos and networks: urbanization and water privatization in the South. Geographical Journal, 169(4), 328-341.

BAкKer, K. (2010): Privatizing water: governance failure and the world's urban water crisis. Cornell University Press, Ithaka NY.

Becker, M. (2012): Building a Plurinational Ecuador: Complications and Contradictions. Socialism and Democracy, 26(3), 72-92.

Boelens, R. (2015a): Water Justice in Latin America. The Politics of Difference, Equality, and Indifference. CEDLA/University of Amsterdam, Amsterdam.

Boelens, R. (2015b): Water, Power and Identity: The Cultural Politics of Water in the Andes. Routledge, Washington D.C., London.

Boelens, R., Hoogesteger, J. y Baud, M. (2015): Water reform governmentality in Ecuador: neoliberalism, centralization and the restraining of polycentric authority and community rule-making. Geoforum, 64, 281-291, doi: 10.1016/j.geoforum.2013.07.005.

ERensu, S. (2013): Abundance and scarcity amidst the crisis of «modern water»: the changing water-energy nexus in Turkey. In L. Harris, J. Goldin y C. Sneddon (Eds.): Contemporary Water Governance in the Global South: Scarcity, Marketization and Participation (pp. 61-78). Routledge, New York.

FERRín-Schettini, R. (1986): Economías campesinas, estructura agraria y formas de acumulación: el caso de Manabí a partir de la Revolución Liberal. PUCE-CONUEP.

Gudynas, E. y Acosta, A. (2010): Si eres tan progresista? por qué destruyes la naturaleza? Neoextractivismo, izquierda y alternativas. Ecuador Debate, 79(5), pp. 61-82.

Hidalgo, J. P., Boelens, R. y Vos, J. (2017): De-colonizing water. Dispossession, water insecurity, and Indigenous claims for resources, authority, and territory. Water History, 9, 67-85, doi: 10.1007/ s12685-016-0186-6.

Hidalgo-Bastidas, J. P., Boelens, R., y Isch, E. (2018): Hydro-territorial configuration and confrontation. The Daule-Peripa Multipurpose Hydraulic Scheme in coastal Ecuador. Latin American Research Review, 53(3), 517-534, doi: https://doi.org/10.25222/larr.362.

Hommes, L. y Boelens, R. (2017): Urbanizing rural waters: Rural-urban water transfers and the reconfiguration of hydrosocial territories in Lima. Political Geography, 57, 71-80.

Hommes, L. y Boelens, R. (2018): From natural flow to «working river»: hydropower development, modernity and socio-territorial transformations in Lima's Rímac watershed. Journal of Historical Geography, 62, 85-95, doi: 10.1016/j.jhg.2018.04.001. 
Hoogendam, P. y Boelens, R. (2018). Dams and Damages. Conflicting Epistemological Frameworks and Interests Concerning «Compensation» for the Misicuni Project's Socio-Environmental Impacts in Cochabamba, Bolivia. Water, 2018.

KeIL, R. (2005): Social power and the urbanization of water: flows of power. Retrieved from http://www. tandfonline.com/doi/full/10.1111/j.1467-8306.2005.00492_2.x

Linton, J. y BudDs, J. (2014): The hydrosocial cycle: Defining and mobilizing a relational-dialectical approach to water. Geoforum, 57, 170-180.

Lynch, B. (2013): River of Contention: Scarcity Discourse and Water Competition in Highland Peru. Journal of International E Comparative Law, 42 (69), 69-92.

Mena-VÁsconez, P., Boelens, R. y Vos, J. (2016): Food or flowers? Contested transformations of community food security and water use priorities under new legal and market regimes in Ecuador's highlands. Journal of Rural Studies, 44, 227-238, https://doi.org/10.1016/j.jrurstud.2016.02.011.

Nixon, R. (2010): Unimagined communities: developmental refugees, megadams and monumental modernity. New Formations, 69(1), 62-80.

Osti, G. (2017): The Anti-Flood Detention Basin Projects in Northern Italy. New Wine in Old Bottles? Water Alternatives, 10(2), 265-282.

Perreault, T. (2014): What kind of governance for what kind of equity? Towards a theorization of justice in water governance. Water International, 39(2), 233-245.

Riaz, K. (2002): Tackling the issue of rural-urban water transfers in the Ta'iz region, Yemen. In Natural Resources Forum, 26, 89-100.

Rodríguez de Francisco, J. C. y Boelens, R. (2015): Payment for Environmental Services: mobilising an epistemic community to construct dominant policy. Environmental Politics, 24(3), 481-500.

Scott, J. C. (1985). Weapons of the weak: everyday forms of peasant resistance. Yale University Press, New Haven, London.

SENAGUA (2012): Informe detallado de los puntos solicitados por la procuraduría general del estado para la defensa del Estado ecuatoriano ante la CIDH. SENAGUA, Quito.

Shah, E., J.W. Liebrand, J. Vos, G.J. Veldwisch y R. Boelens (2018): The UN World Water Development Report 2016, Water and Jobs: A Critical Review. Development and Change, 49(2), 678-691, doi: $10.1111 /$ dech.12395.

Swyngedouw, E. (1995): The contradictions of urban water provision: a study of Guayaquil, Ecuador. Third World Planning Review, 17(4), 387-406.

Swyngedouw, E. (1997): Power, nature, and the city. The conquest of water and the political ecology of urbanization in Guayaquil, Ecuador: 1880-1990. Environment and Planning A, 29, 311-332.

Swyngedouw, E. (2004): Social power and the urbanization of water: flows of power. Oxford University Press, Oxford.

Swyngedouw, E. (2015): Liquid Power: Contested Hydro-Modernities in Twentieth-Century Spain. MIT Press, Cambridge MA. 
Valladares, C., y Boelens, R. (2017): Extractivism and the rights of nature: governmentality, «convenient communities», and epistemic pacts in Ecuador. Environmental Politics, 26(6), 1015-1034, doi: 10.1080/09644016.2017.1338384.

Van Teijlingen, K. (2016): The «will to improve» at the mining frontier: Neo-extractivism, development and governmentality in the Ecuadorian Amazon. The Extractive Industries and Society. 3(4), 902-911.

Vojinovic, Z. (2015): Flood Risk: The Holistic Perspective. IWA Publishing, Londres.

Watkins, K., Carvajal, L., Coppard, D., Fuentes, R., Ghosh, A., y Giamberardini, C. (2006): Human Development Report 2006: beyond scarcity: power, poverty and the global water crisis. United Nations Development Programme (UNDP), Rome.

Zwarteveen, M. (2015): Regulating water, ordering society: practices and politics of water governance. Universiteit van Amsterdam, Amstterdam.

Zwarteveen, M., y Boelens, R. (2014): Defining, researching and struggling for water justice: Some conceptual building blocks for research and action. Water International, 39(2), 143-158, doi:10.10 80/02508060.2014.891168.

Cómo citar este artículo:

Hidalgo-Bastidas, J.P. y Boelens, R. (2018). Inundaciones políticamente construidas.

El megaproyecto hídrico Chone en Ecuador. Cuadernos de Geografía, 101, 127-148.

https://doi.org/10.7203/CGUV.101.13724

\section{(C) $(1) \Theta \Theta$}

Este obra está bajo una licencia de Creative Commons Reconocimiento-NoComercial-SinObraDerivada 4.0 Internacional. 
